\title{
The Online Poker Sub-Culture: Dialogues, Interactions and Networks
}

\begin{abstract}
This paper examines the distinct world of online poker. It outlines the online poker ecosystem, and the players which inhabit it, their distinctive attitudes and behaviors towards the game and gambling. These unique patterns of behavior have been created and sustained by the interaction of players within online poker forums. Therefore online poker forums were identified as primary mechanism within which a poker sub-culture may exist. The study conducted an extensive netnography of online poker forums. The study found that within the online poker eco-system there are forums which are elevated to sacred status amongst this online poker sub-culture. The members of these forums enact sub-cultural characteristics such as ethos of collaboration/ cooperation, and a competitive hierarchy of status. In particular this paper identifies the importance of identity generation, and communitas within the online poker eco-system.
\end{abstract}

\section{Keywords}

Online Poker, Subculture, Virtual Community, Virtual Identities, Netnography, Gambling 


\section{The Online Poker Sub-Culture: Dialogues, Interactions and Networks}

\section{Introduction}

Over the past decade a complete online poker eco-system has evolved online where individuals gamble, interact, network and consume regularly. With the advent of the digital technological revolution, the study of online communities has been of increasing focus within academia (see Kozinets 1997 \& 2001, Muniz \& O’Guinn 2001, Muinz \& Schau 2005, Schau et al 2009). The purpose of this paper is to provide an insight into some of the characteristics of the online poker world building upon the extensive works of Parke \& Griffiths (2011), Griffiths et al. (2010), McCormack \& Griffiths (2012a), McCormack \& Griffiths (2012b), Parke \& Griffiths (2012), Mitrovic \& Brown (2009), Siler (2010), Wood et al. (2007a), La Plante et al. (2009), and Hopley \& Nicki (2010) The paper will demonstrate firstly the importance of online poker forums to this virtual world, then moving on to illustrate some of the key characteristics demonstrated by what we coin the Online Poker Subculture (OPS) namely: collaboration/co-operation and hierarchy. From this a detailed analysis of the implications of these characteristics will be discussed to further work on the pivotal role that community and identity play within the OPS.

Online poker has dramatically altered the shape of gambling since its inception in 1998 when the first online poker site, Paradise Poker was launched. Conversely online poker 
has recently been faced with its biggest challenge to date. The US government has initiated legal frameworks banning US citizens from playing online poker. In 2006 the US Congress decreed that online gambling was illegal within the US, with the Unlawful Internet Gambling Enforcement Act (UIGEA); however sites such as FullTiltPoker.com and PartyPoker.com still facilitated online play for US players. These sites and many others avoided prosecution for several years through a legal loophole until the US government eventually shut down their operations on the 20-09-2011, now famously dubbed as "Black Friday" within poker parlance.

Online poker has evolved to a point where there are now a variety of facets to its existence, which have been duly created by and for the community of players who inhabit this world. The online-poker ecosystem can be categorized into four distinct platforms that players frequent, consuming and exchanging their passion for the game; Online Poker sites, Online Poker forums (e.g. 2+2), Player Reporting/Tracking sites (e.g. PokertableRatings) and Popular magazine/news sites (e.g. Cardplayer, Bluff, Pokernews.com) as illustrated in Table 1.

(Insert Table 1) 


\section{Theoretical Overview}

Over the years research has been fascinated by groups/groupings of people (Cova \& Cova 2001, Celsi et al. 1993, Johnson 2001, Horrigan et al. 2002) be it from an anthropological, philosophical or a behavioral standpoint. Subcultural studies have furthered our understanding of devout groups of people immersed within specific committed intensive environments. Celsi et al. (1993) have outlined the rites of passage that subcultures enact. Schouten and McAlexander (1995) have illustrated the hierarchy and ethos within the Harley Davidson subculture and more recently Cova \& Cova (2001) have theorized on the social and communal links formed within subcultures.

Kozinets has pioneered studies of fan-based subcultures and how their communication over the Internet affects subcultural characteristics (Kozinets 1997, 2001). Other studies too have laid the foundations for our understandings of online groups (see Johnson 2001, Horrigan 2002, Wilson and Peterson 2002). One online grouping that has been growing on an exponential scale over the past ten years is online poker; see McCormack \& Griffiths (2012a). With the ever increasing rise of online poker into popular culture advocated through advertising and product placement, embedded within film and television entertainment, the number of people now partaking in online poker play is astounding, with Parke \& Griffiths (2011:31) outlining that "internationally the prevalence of online poker gambling is estimated to be between $1 \%$ and $8 \%$ of the general adult population". 
Academic work in recent years with reference to the study of online gambling and in particular online poker has been gaining momentum. Wood et al. (2007a) in their study of online poker play within a student sample illustrated the various motivators for participation and highlighted the predictive signs of problematic online gambling. Other studies too have looked at the motivations behind online poker play and the development of corresponding problematic gambling behavior (see McCormack \& Griffiths 2012b, Wood et al. 2007a, McBride \& Derevensky 2009, Hopley \& Nicki 2010, and Mitrovic \& Brown 2009). Khazaal et al. (2011) has examined online poker sites and their pathological gambling prevention strategy, and furthermore there has been extensive work by Siler (2010) on online poker gambling patterns, where gamblers utilise complex psychological and sociological factors in making their risk decisions. Building on these works further studies have illustrated the behavioral aspects of online poker play (Griffiths et al 2010, McCormack \& Griffiths 2012a \& LaPlante et al. 2009), and how the use of information technology has enamored such stringent profitable play within online poker (Parke \& Griffiths 2011 \& Parke \& Griffiths 2012).

However this paper will focus on the subculture which exists within the online poker ecosystem, the online poker subculture (OPS), namely those active within online poker fora. We propose that online poker forums are home to those immersed within the OPS and contend that evidence gained from an ethnographic/netnographic study of this subculture could prove invaluable to the online poker and gambling industry. Schultz (2000) in his study of online forums and their interactive properties states that there are a growing number of communication and information forums now available to consumers. $\mathrm{He}$ 
contends that they help reduce complex problem solving, (i.e. in this case relevant to advancing one's poker game skills), while also facilitating the rise of a shared beliefs system (in online poker's case a subculture).

Building upon this and in particular McCormack \& Griffiths (2012b) grounded theory surrounding online poker play we aim to introduce the concept that identity and communitas are integral to poker playing motivations. The work in particular of McCormack \& Griffiths (2012a) and Parke \& Griffiths (2011) has hence expanded our viewing of online poker play/ers and this paper we hope will enamor further theoretical insight into this online grouping. McCormack \& Griffiths (2012b) grounded theory on the motivating and inhabiting factors surrounding online gambling has proved seminal in provided scholars with a basis to understand the dynamics of online gambling participation.

The work of Mitrovic \& Brown (2009) building on the self-determination theory of Deci (1971) \& Ryan \& Deci (2000) has outlined two of the main motivating factors in gambling online, developing from two psychological needs: that of autonomy and competence. We advocate this dichotomy as Mitrovic \& Brown (2009:491) note that when "competence is rewarded it encourages intrinsic motivations". For us these intrinsic motivations within online poker gambling are much more than that of simply monetary gain through the development of poker skills (Parke \& Griffiths 2011), convenience, greater variety of games online, better value for money or a safe world (McCormack \& Griffiths 2012b), to that of a search for communitas and a sense of 
communal identity through online poker forums and the creation of a "known" online identity within the poker world. McCormack \& Griffiths (2012b:50) contest that "the medium of online gambling can also lead to a reduced authenticity of gambling and thus inhibiting some individuals from gambling online" and while this may be congruent of other forms of online gambling we contest that online poker does not support this theory. Thus motivating factors for participation within the OPS are more complex and differ greatly from other gambling contexts. The paper highlights two subcultural characteristics, that of collaboration/co-operation and hierarchy which reinforces our stance as to why communitas and identity are central to online poker playing motivations. Past studies such as Wood et al. (2007b) have identified several motivational factors within online gambling contexts, yet there is a gap in the examination of the role of communitas, and identity creation within online poker.

Similarly the role of identity needs to be addressed and its relevance to online poker. We propose that online poker fora allow participants to create and mold an online persona by the exposure of successful online play and adherence to forum participation etiquette. Parke \& Griffiths (2011) have outlined how experiential reporting is central to developing cognitive poker skills through social reinforcement and extricating frustration. Individual motivations to play poker and participate in online forums are driven by a want/desire to become recognized by one's peers leading to the creation of a peer recognised persona within the OPS. This identity creation however is warranted by the hierarchy within the OPS and our aim is to expose some of the procedures which are 
enacted in the process of successful identity creation within the OPS for hardcore/avid players.

\section{Method}

For the purpose of this paper it was decided by the authors to conduct an in-depth qualitative analysis of the online poker world. The methodology utilized was netnography. The study was conducted over an 18 month period of immersion and a subsequent 6 month data collection period by one of the authors into three major online poker forums in conjunction with observation of the online poker ecosystem platforms already outlined. Kozinets (2002) has stated that in recent years there have been calls from many anthropologists, sociologists and business researchers to adapt existing ethnographic research techniques to the many cultures and communities that are emerging through online communications. Thus netnography then is an extension of ethnography and as Kozinets (2002:62) outlines "Netnography or ethnography on the Internet is a new qualitative research methodology that adapts ethnographic research techniques to study the cultures and communities that are emerging through computermediated communications".

Yuan et al (2007) also outlines that large-scale communities offer great environments to study the social and economical behavior of large populations as they can be accessed with ease while the pseudo-anonymous nature of the online environment encourages users to display their true behaviors. As Griffiths (2010:12) notes "some of the most interactive and textually rich parts of the internet are numerous gambling and gaming 
forums". Another key influencing factor in our decision to pursue netnography as a viable and credible methodology was that its unobtrusive nature allowed us to view online poker participants in their natural setting untainted by the presence of a researcher (Tulloch \& Jenkins 1995:281-282 cited in Kozinets, 1997, Griffiths 2010). Wood \& Griffiths (2007b) note that data within gambling forums can be collected without identifying oneself as a researcher. Spradley (1980) outlines this as critical to avoiding research bias when operating within gambling contexts. Furthermore Kozinets (2002) goes on to state that online communities chosen for a netnographic study should have a high traffic of postings, more detailed or descriptively rich data and a large amount of between member interactions. Thus Netnography is a highly appropriate methodology for this field of enquiry in order to verify if a subculture existed within the online poker world, and if so to identify the subcultural characteristics its members displayed and if these differed or were comparable to previous studies.

Participant observation/lurking was the main method employed to collect data. This presented the authors with some concerns with reference to the study of online poker forums. As Griffiths (2010) notes when studying online forums there is a debate between what is public and private knowledge. The issue then of privacy as noted by Wood \& Griffiths (2007b) had to be addressed. As Griffiths \& Whitty (2010:111) note "the general rule of thumb is that researchers should only observe people in a situation where they would ordinarily expect to be observed". Therefore any and all netnographic dialogue collected was solely relating to gameplay and not in any way associated with ethical issues such as problem gambling etc. The netnography is based on the observation 
of low/medium/high stakes game play threads specifically, as these threads seek information from the public population of the forums. Other sections such of that of BBV (Brags, Beats \& Variance) within $2+2$ were not subject to this study where issues such as addictive and problematic gambling, gaming and forum use are readily discussed.

\section{Procedure}

Data was collected over six months subsequent to the 18 months immersion period. In total three online poker forums were subject to netnographic investigation. These forums were chosen on the basis of popularity and continuous interface within the online poker world, having each a substantial number of active participants. The three forums studied were Deuces Cracked (Membership $=90,000)$, High Stakes Database (Membership = 60,000), and 2+2 (Membership = 320,000). Access to these forums was gained by providing an email address, username and password. Once the author received access to each of the forums the immersion period of 18 months was spent learning the language, rituals, and etiquette of these fora. From this a data collection period of 6 months was enacted. Game play sections of the three chosen forums were observed daily by the author. Threads deemed to be worthy of investigation based on the research objectives were then subsequently subscribed to. This enabled the researcher to track and archive player interactions, and behaviors, for later content analysis which Griffiths (2010) states allows for time based qualitative research to be conducted. Subsequent to this data collection period the method of test-retest reliability was employed by the second author to ensure consistency across the qualitative content analysis conducted by the first researcher. Any inconsistencies were highlighted and evaluated thus prompting further 
qualitative investigation by the first researcher. This process was enacted on a number of occasions throughout the research process.

\section{Results}

On completion of the analysis it was evident, firstly that the OPS did exist from the display of subcultural characteristics. These characteristics included; an ethos of collaboration/cooperation, and a competitive hierarchy of status. From this our analysis highlighted that the forum $2+2$ was a sacred place to the OPS inhabited by hardcore/avid participants. The research yielded insight into the distinct online poker language, and online gameplay which has transformed offline poker. In addition the enactment of the ethos of the OPS served the dual process of generating a sense of communitas, and peer recognition. The authors uncovered an ethos of cooperation/collaboration - which enamors a motivation to partake in online poker forums. Online poker's image is seen as highly unsocial (McCormack \& Griffiths 2012b); however these forums act as an outlet for intense social interactions, and dialogues surrounding the game. Furthermore these social interactions are governed by a distinct hierarchy within the OPS. Through the enactment of the ethos, player personas are generated which are aligned to; online poker play; quantity and quality of forum participation; and adherence to forum norms.

Online poker forums have become "sacred temples" to the OPS because they replace the need for contact/shared experiences in an offline world and give rise to social ties in an online world. These online members avidly post their thoughts, interact, observe and enact their cultural characteristics (OPS) within these online poker forums, enabling it to gain a sacred status among members of the subculture. Online poker forums allow 
players to develop their own online persona, through interaction, participation and engagement with the subculture, thus reaffirming their reputation amongst their poker peers

These forums influence upon the context of offline poker has revolutionized the game. Elements such as online poker language, betting styles (see figure 1), strategies, and mathematical based play terms (e.g. VPIP\%, PFR\%, Fold Equity, All in EV) have all transcended into the offline game changing the very nature in which the game of poker is now played (see figure 1). Online sub-cultural characteristics of online poker have traversed into the traditional poker gaming, which is evidenced through gaming play, lexicon, poker gambling participation, and the lack social interaction/dialogue during physical game play. Forums have become sacred and integral to the OPS because they provide the medium for the subculture to enact their subcultural norms and patterns of behavior. The sacred status of $2+2$ is thus validated by numerous success stories relating to their members along with the increasing adoption of its ideology into the offline poker world.

\section{(Insert Figure 1)}

The ethos of the subculture serves as a means of furthering the progression, profitability and success of its membership. Social ties within the subculture are enhanced through the exchange of information, creating a sense of goodwill/communitas among members. Below is a post where a member seeks advice from the OPS on how to play a hand against an unknown player. This demonstrates how the OPS and the forums they inhabit 
exude the ethos of co-operation/collaboration, which is in alignment with the findings of Parke \& Griffiths (2011), which emphasize experiential reporting. Members who divulge key information beneficial to the group are often praised, resulting in relationship formation. This exchange enhances the status of the player/s within subculture. A member enacting the ethos thus can take steps to rising the hierarchy of the subculture. This illustrates the importance of communitas to the OPS, as it facilitates persona generation through the enactment of the ethos, while simultaneously aiding in developing cognitive poker skills among members. Those members who achieve recognition for their contributions are lauded by their peers.

\section{(Insert Figure 2)}

The enactment of the ethos serves as a platform to enhance players' online identities, which is central to rising within the OPS hierarchy. The OPS may exist in a virtual world but has many of the same traits as an offline subculture. Past studies such as Schouten and McAlexander (1995), Thorton (1995), Fox (1987) and Kates (2002) have identified that subcultures have a distinct hierarchy in place within their group, with observed rules, rituals, and norms commonplace to subcultural existence. The same ideal is in place within the OPS where the hierarchy and ultimately those at the top of the spectrum set the guidelines for identity creation, conformity and substance. The post below is an example of this rule setting by members of the hierarchy. The original poster has displayed a hand he had previously played online and wanted to seek advice on how to approach the hand. 
The respondents replied with how the player should have played and conformed to the optimal situational play deemed appropriate by the OPS hierarchy.

(Insert Figure 3)

It is clear then that members of the OPS in order to be accredited must adhere to the hierarchy and conform to the views of the collective. It is integral to each member's status that they enact and adhere to the ideals and ethos of the OPS as members are and have been in the past ostracized for non-conformity. Another facet of the hierarchy which governs identity creation of members of the OPS is the level of engagement of members within poker forums. The more one engages and participates in online forums the higher they are elevated within the subcultures hierarchy, while also in turn gaining recognition for their online identity. This is done in the OPS to such an extent that a member's activity/frequency on online forums has become an integral part of one's identity. Indeed in most online forums members are assigned statuses based on their frequency/activity and number of postings. One prime example of this is in online forum $2+2$ where members are given different labels based on the number of posts they have made (see figure 4).

\section{(Insert figure 4)}

Online forum member's identities also display their join date (i.e. the date they joined the site). This is also a sign of status as those who have been members since the inception of these forums are viewed as elevated members and pioneers of the subculture. Given 
below is an example of this where a "newbie" in his first post in an online forum is attacked for posting an online hand of Omaha type poker he recently played and won. In fact "Respondent $E$ " was a newbie also but had a higher volume of posts and saw an opportunity to gain credibility by other forums users, in turn enhancing his own online identity and making strides to rise the OPS hierarchy through flaming, which Kozinets (1997:471) outlines is the practice of sending embarrassing and insulting e-mail messages to a person's mailbox or a negative response to their public forum posting.

\section{(Insert Figure 5)}

Using Kozinets (2002) typology of forum members the OPS can be classified into three distinct groups; Devotees, Minglers and Insiders. However a fourth group has been identified within the OPS, and we have labeled this group as Lurkers. Lurkers are members, which have a high interest in the activity of the OPS, but are unwilling or even fearful to form social ties to the group for risk of flaming. The OPS have also developed an extension or new variety of flaming, it involves insulting people through being highly sarcastic in their replies to posts by trepid lurkers or junior members, which the OPS terms as 'Leveling'. It is clear from this then that members of the OPS must adhere to the hierarchy which is in place and the elements of conformity they enact and emplace upon the group or risk flaming or leveling as a result. This hierarchy thus governs identity creation of online player/s through the avocation all members enact an ethos of collaboration/co-operation whilst conforming to the norms of OPS etiquette. 


\section{Discussion}

\section{"The Sacred Temple" - The World of Online Poker Forums}

Previous studies on subcultures have exposed how they can become central to members identity and existence, very often the context becoming elevated to a sacred status within their membership (Schouten and McAlexander 1995, Thornton, 1995, Celsi et al. 1993, Cova \& Cova 2001, Chalmers \& Arthur 2008). Within the OPS the forum $2+2$ has been elevated to this scared status. It has gained this status among members as it is primarily their place, unadulterated and untainted by external stakeholders.

Aiding in this elevation of $2+2$ to that of sacred status among the OPS is the fact that these forums has given birth to online poker celebrities. A key point however is that these players molded an online identity through both successful online play and intense interaction on $2+2$. Both factors are critical in constructing an accredited online identity which we believe is a key motivating factor to interacting on poker fora and ultimately playing online poker. Many newbies join forums such as $2+2$ to try and emulate their success. Therefore the implication is that to become a highly successful online poker player and to receive accreditation, monetary results are not solely sufficient. Each successful player must have constructed an accredited identity through the enactment of the ethos of the OPS while adhering to its hierarchy.

Therefore these forums become sacred temples/places to those immersed within the ranks of this online culture. They allow members of the OPS to interact with one another frequently, enact status, and to ultimately garner recognition and reputation of their 
online identities. A shared beliefs system gives rise to a hierarchy, while problem solving is vital to the ethos of the OPS. Forming a mutual identity serves to differentiate the online poker world and in turn leads to culture creation for the offline poker world (See appendix 1 for the language used within the OPS). Thus far academic work surrounding online poker has not highlighted the extraordinary influence of online poker on changing the way the game is played in an offline context. Similar online forums have also been elevated to such a status, such as Magicseaweed.com for the surfing sub-culture, or Ultimateguitar.com for guitar aficionados. Gambling forums such as OLBG.com, and therxforum.com are actively seeking to emulate this sacred status for gamblers, with huge virtual communities actively engaged in dialogues surrounding gambling.

\section{"Don't Bet into the Donkey" - Collaboration/Cooperation in the OPS}

As Schouten and McAlexander (1995:43) state the characteristics of a subculture include “an identifiable, hierarchical social structure, a unique ethos, or set of shared beliefs and values; and unique jargon, rituals and modes of symbolic expression". The OPS is governed by a set of common values (ethos) among members which hold an extraordinarily amount of weight and history within the group. An ethos of cooperation and collaboration evolved from players seeking a competitive advantage over those not immersed within the OPS. This is achieved primarily by offering advice to other forum members, seeking advice (e.g. posting hands one has played on forums and seeking advice on optimum levels of play) and offering any valuable information to the community as a whole (e.g. where there are easy games, or creating optimum strategies for various poker games/formats). This represents an interesting dichotomy between the competitive nature of the 'individual' and the collaboration within the 'collective'. 
Yuan et al (2007:475) has noticed that "co-operation can emerge as a norm in an environment comprising individually selfish entities". This is an interesting concept as primarily the game of poker is an individual occurrence but the OPS bands together to enhance their poker skills through cooperation and collaboration. Parke \& Griffiths (2011) have already made reference to this in their study use of computer mediated communication in developing cognitive poker skills. However we believe the use of online poker forums within the OPS is a myriad of complex motivations driven by its ethos. Firstly members seek to develop cognitive poker skills through these forums to become profitable from those not involved in the subculture. This dually leads to both monetary gains and an individual's online identity being recognized. From this a sense of communitas is established which defines the OPS and sustains the forum's sacred status to those who inhabit it.

This research has opened up a multitude of research questions within the area. More work is needed on the creation and sustainment of an online identity. Further research on how the offline poker community perceives this new breed of online poker players is required, to ascertain commonalities and/or differences between both groups, building on the work of McCormack \& Griffiths (2012a). While this paper has identified the ethos and hierarchy of the OPS, further analysis is needed to examine areas such as the use of online poker forums to address problematic gambling behavior, and motivations behind addictive gaming. Forums can potentially act as a safety net in influencing responsible 
gaming and gambling (Parke \& Griffiths 2011) through the enforcement of its ethos and stringent behavioral norms (Parke \& Griffiths 2012) relating to online poker play.

\section{"How did you call that bet?" - Competition and Hierarchy within the OPS}

The OPS hierarchy is formed solely in a virtual world. Therefore there are different implications for the role of the hierarchy within the OPS as opposed to those of an offline subculture. The hierarchy of the OPS is primarily based on the frequency of interaction with the other members via forums (i.e. the number of posting one engages with), results of one's online poker capability (which can be tracked via Pokertableratings.com/ Sharkscope), and the enactment of the subculture's ethos. If these factors are successfully negotiated then recognition and reputation (two elements pivotal to hierarchical status) are enhanced for members.

It has been noted in this paper that the hierarchy within the subculture governs the level of usage. Opinion leaders shape the terms and conditions of usage of their peer groups within the sub-culture as is demonstrated in figure 4 . This can potentially lead to problematic gambling activity as noted by Parke \& Griffiths (2011) and also excessive forum usage through the avocation of constant participation and involvement. As a result of this there are implications for making interventions surrounding health promotions in addressing gambling addictions within the subculture. Health promotion advocates need to understand these online communities, engage with them, and potentially leverage their position within the hierarchy. This will enable health promotion advocates to create more effective social responsible gambling campaigns. Failure to do so represents a lost opportunity in addressing the negative societal dimension to gambling. 


\section{Limitations}

It is important to note that this study is not without its limitations. As Griffiths \& Whitty (2010) outline some of the main areas of concern related to online data collection are: lack of knowledge about participant behavior and validity. While the test-retest method to ensure reliability in the data was employed the nature of the study afforded us no opportunity to gain an insight into how online poker affects individuals in an offline context. Future studies could contrast the impact of excessive or problematic gaming, gambling and forum use on individual motivations and identities within an offline context. We must also acknowledge that the study has had a sampling bias towards that of members who engage within gameplay specific threads. Further investigation into the totality of threads within online poker forums may offer some new insights be they into subcultural characteristics of the OPS, gambling motivations or predictors of problem gambling/forum use.

\section{Conclusion}

The paper thus far has established that there is a subculture which exists within the online poker world and in turn there are huge commercial implications for the online gambling poker industry and future studies relating to online poker activities. Firstly the medium of online poker forums while catered to has not truly been exploited by commercial interests within the poker industry. Through understanding the OPS one can ascertain key insights into these gamblers. Members display their current moods, wants, and desires, which organizations can use to pre-empt or react to trends within the OPS. Secondly the OPS ecosystem provides rich detailed information which researchers have failed to capitalize 
on convincingly. Future studies surrounding online poker should be acquainted with this grouping and research efforts must be made to address the extreme levels of consumption practices the OPS enacts.

Netnography can provide a huge insight into attitudes, and patterns of gambling behavior, keeping abreast of a rapidly changing environment by tracking conversations/threads of online poker forums. This knowledge informs us as to where online players gamble, who online players talk to, what online players read, what online players search for, and above all their elusive patterns of behaviour.

This paper has illustrated the development of online poker forums into achieving scared status for avid online poker players. This status is sustained through rich online dialogue, interaction, and network formation. This world distinctively enacts a contradiction, in that within a context of individually driven selfish motives (i.e. everyone playing to win), collaboration and cooperation comes to the fore within the OPS. However throughout the OPS there still remains fundamental competition in the attainment of recognition and development of individual online identities through the OPS hierarchy. The OPS has made a huge impact on the poker world, in effect creating and developing an expanding online poker eco-system, whilst transforming the context the traditional offline poker universe. 


\section{References}

Celsi, R. L., Rose, R. L., \& Leigh T. W. (1993). An exploration of high-risk consumption through skydiving. Journal of Consumer Research, 20, 1-23.

Chalmers, T. D., \& Arthur, D. (2008). Hard-core members of consumption-orientated subcultures enactment of identity: The sacred consumption of two subcultures. Advances in Consumer Research, 35, 570-575.

Cova, B., \& Cova, V. (2001). Tribal aspects of postmodern consumption research: The case of French in-line roller skaters. Journal of Consumer Behaviour, 1, 67-76.

Deci, E. L. (1971). Effects of externally mediated rewards on intrinsic motivation. Journal of Personality and Social Psychology, 18(1), 105-115.

Fox, K. J. (1987). Real punks and pretenders: the social organization of a counterculture. Journal of Contemporary Ethnography, 16, 344-370.

Griffiths, M. D. (2010). The use of online methodologies in data collection for gambling and gaming addictions. International Journal of Mental Health and Addiction, 8, 8-20. 
Griffiths, M.D., Parke, J., Wood, R., \& Rigbye, J. (2010). Online poker gambling in university students: Further findings from an online survey. International Journal of Mental Health and Addiction, 8, 82-89.

Griffiths, M. D., \& Whitty, M. W. (2010). Online behavioural tracking in Internet gambling research: ethical and methodological issues. International Journal of Internet Research Ethics, 3, 104-117.

Hopley, A. A. B., \& Nicki, R. M. (2010). Predictive factors of excessive online poker playing. Cyberpsychology, Behavior \& Social Networking, 13, 379-385.

Horrigan, J. B., Rainie, L. \& Fox, S. (2002). Online communities: networks that nurture long-distance relationships and local ties. Connecticut, Washington: Pew Internet and American Life Project.

Johnson, C. M. (2001). A survey of current research on online communities of practice. Internet and Higher Education, 4, 45-60.

Kates, S. M. (2002). The protean quality of subcultural consumption: An ethnographic account of gay consumers. Journal of Consumer Research, 29, 383-399. 
Khazaal, Y., Chatton, A., Bouvard, A., Khiari, H., Achab, S, \& Zullino, D. (2011). Internet poker websites and pathological gambling prevention policy. Journal of Gambling Studies, 28, 1-9.

Kozinets, R. (1997). I want to believe: a netnography of the x-philes' subculture of consumption. Advances in Consumer Research, 24, 470-475.

Kozinets, R. (2002). The field behind the screen: Using netnography for marketing Research in Online Communications. Journal of Marketing Research, 39, 61-72.

Kozinets, R. (2001). Utopian enterprise: Articulating the meanings of star trek's culture of consumption. Journal of Consumer Research, 28, 67-88.

LaPlante, D. A., Kleschinsky, J. H., LaBrie, R. A., Nelson, S. E., \& Shaffer, H. J. (2009). Sitting at the virtual poker table: A prospective epidemiological study of actual Internet poker gambling behavior. Computers in Human Behavior, 25, 711-717.

McBride, J. \& Derevensky, J. (2009). Internet gambling behaviour in a sample of online gamblers. International Journal of Mental Health and Addiction, 7, 149-167.

McCormack. A. \& Griffiths, M. D. (2012a). What differentiates professional poker players from recreational poker players? A qualitative interview study. International Journal of Mental Health and Addiction, 10, 243-257. 
McCormack, A. \& Griffiths, M. D. (2012b). Motivating and inhibiting factors in online gambling behaviour: A grounded theory study. International Journal of Mental Health and Addiction, 10, 39-53.

Mitrovic, D. V. \& Brown, J. (2009). Poker mania and problem gambling: A study of distorted cognitions, motivation and alexithymia. Journal of Gambling Studies, 25, 489502.

Muinz, A. M. \& O'Guinn, T. C. (2001). Brand Community. Journal of Consumer Research, 27(4), 412-432.

Muinz, A. M. \& Schau, H. J. (2005). Religiosity in the abandoned apple newton brand community. Journal of Consumer Research 31(4), 737-747.

Parke, A. \& Griffiths, M.D. (2012). Beyond illusion of control: An interpretative phenomenological analysis of gambling in the context of information technology. Addiction Research and Theory, 20, 250-260.

Parke, A. \& Griffiths, M. D. (2011). Poker gambling virtual communities: The use of Computer-Mediated Communication to develop cognitive poker gambling skills. International Journal of Cyber Behavior, Psychology and Learning, 1, 31-44. 
Ryan, R. M., \& Deci, E. L. (2000). Intrinsic and extrinsic motivations: Classic definitions and new directions. Contemporary Educational Psychology, 25, 54-67.

Schau, H. J., Muinz, A. M. \& Arnould E. J. (2009). How brand communities practices create value. Journal of Marketing 73(5), 30-51.

Schouten, J., \& McAlexander, J. (1995). Subcultures of consumption: An ethnography of the new bikers. Journal of Consumer Research, 22, 43-61.

Schultz, T. (2000). Mass media and the concept of interactivity: An exploratory study of online forums and reader e-mail. Media, Culture \& Society, 22, 205-221.

Siler, K. (2010). Social and psychological challenges of poker. Journal of Gambling Studies, 26, 401-420

Spradley, J. P. (1980). Participant Observation. New York: Holt: Rinehart \& Winston.

Thornton, S. (1995). Club culture. Cambridge, UK: Polity Press.

Tulloch, J. \& Jenkins, H. (1995). Science fiction audiences. New York: Routledge.

Wilson, S. M., \& Peterson, L. C. (2002). The anthropology of online communities. Annual Review of Anthropology, 31, 449-467. 
Wood, R. T. A., Griffiths, M. \& Parke, J. (2007a). Acquisition, development, and maintenance of online poker playing in a student sample. CyberPsychology \& Behavior, $10,354-361$.

Wood, R. T. A. \& Griffiths, M. D. (2007b). Online data collection from gamblers:

Methodological issues. International Journal of Mental Health and Addiction, 5, 151163.

Yuan, R., Zhao, L. \& Wang, W. (2007). Cooperation and competition dynamics in an online game community. Online Communities and Social Computing, 4564, 475-484. 


\section{Appendix 1}

\section{The Language of the OPS}

Terminology

A - Ace

K - King

Q - Queen

J - Jack

$\mathbf{T}-$ Ten

TP - Top Pair

NL - No Limit

FL - Fixed Limit

Fish - Refers to a bad player who is relatively easy to beat and outplay

Donkey - A term used to describe an extremely poor player

Luckbox - A name often given to players who get lucky in a specific hand

Shark - A good player of online poker whose skills are far superior of that than of recreational players e.g. fish

SB (Small Blind) - Forced bet which is made by the player sitting to the dealer's left.

BB (Big Blind) - Forced bet before the hand starts it is always double the small blind. The player to the left of the small blind has to post it before the hand starts.

UTG - The person first to act in a hand i.e. the person to the direct left of the big blind.

UTG+1 - The person to the direct left to UTG i.e. the second person to act in a hand.

MP - Middle Position

HJ (HighJack) - The position directly to the cut offs right

CO (Cut Off) - The position directly to the dealer's (button's) right

(BTN) Button - The position of the dealer in a hand of poker. It is the held by the player

to act last after each round of betting. Considered to be a huge advantage when playing poker. It rotates between the players evenly after each hand.

Street - Refers to the different stages of a poker game i.e. first street in hold'em is the flop, $2^{\text {nd }}$ street is the turn and $3^{\text {rd }}$ street is the river. An example of it in use is "bet every street".

Range - Players refer to what type of hands other players can have by using the term range i.e. "his range was AA, KK, QQ"

Villain - When a player is talking about a hand they have played they refer to the opposite player as "Villain"

Hero - When a player is talking about a hand they have played they refer to themselves as "Hero"

LAG (Loose Aggressive Player) - Refers to a player that plays a lot of hands and plays them very aggressively

TAG (Tight Aggressive Player) - Refers to a players that only plays a very small number of premium hands and plays them very aggressively

f/d (Flush draw) - This is when a player have two cards of the same suit in his hand are there are two cards matching his/her suit on the flop/turn and he/she is hoping to make a flush 
C-Bet (Continuation Bet) - Means that a player continues betting if he has raised preflop. E.g. Player one raises to 200, Player two calls. The Flop is King of Hearts, Ace of Club and Two of Diamonds, Player one c-bets 400

Snap call - Means to call a bet instantly

Hero call - Means to make a call on the river with a marginal hand and win the hand i.e. to know when an opponent is bluffing

Flat - Means to call a bet

Floating - Means to call the flop or turn with a poor hand in order to try and bluff later in the hand

Limp - Means to just call the big blind and not raise

OOP (Out of Position) - Means to play a hand where you must act must on the flop/turn and river. This is a distinct disadvantage in poker.

BvB - Refers to Blind versus Blind situations, where only the small and big blinds are involved in a hand.

b/f (bet/fold) - Means to bet your hand but if raised by your opponent to then fold your hand.

c/c (check/call) - Means to check your hand and then call a bet from your opponent

c/r (check/raise) - Means to check your hand and then raise your opponent if they bet. This is usually seen as a sign of extreme strength and aggression but can also be used as a good bluffing tool.

c/f (check/fold) - Means to check your hand and fold to any bet that is made by other player/s.

HU (Heads Up) - Means when there are only two players at a table or involved in a hand HH (Hand History) - Refers to the history of poker hands an online player has played. Very often online players will save their hand history and analyse their play in a critical fashion.

Uber Tilt - Means to be affected by circumstances which affect your thinking in a game of poker E.g. Losing a hand you should have won and then going on "Uber Tilt" and not playing well or to your potential, basically letting your emotions dictate the way you play.

Stacked - Means to take all of another player's chips/money

Dry/Wet Board - Refers to the texture of the flop i.e. if the flop is not co-ordinated (3 different suited cards not connected e.g. Kings of Hearts, Two of Spades and Nine of Diamonds) it is referred to as a Dry Board. If the Board is Wet then it is co-ordinated e.g. Four of Spades, Five of Spades and Six of Diamonds.

Plus/Negative EV (Expected Value) - A complicated poker term that uses maths to determine the expected value a player has in a poker game. If a player has a Plus EV and still loses money in a poker session it would then suggest that he/she was unlucky or variance was against them.

Defending - Means to call a bet when you are one of the blinds and a player raises Line Check - A phrase used to ask if the correct/optimum play was made in a scenario Semi-Bluff - Means to bet when you are drawing to a hand. E.g. when you have two diamonds and there are two diamonds on the flop but your hand has no value unless you make your flush

Combo Draw - This refers to when a player has a draw to both straight and flush possibilities. 
PFR - Refers to the percentage of times a player raises pre-flop. The higher the percentage the more aggressive the player.

ISO - Means to isolate a player in a particular hand by raising out other players if you feel confident that you have the best hand and one particular player will pay you off or that you can outplay that particular player.

Light - Means raising with a less than premium hand and is used in this way "I was 3betting light a lot"

Sit and Go - Refers to a once off tournament type game of poker where each player buys in for a set amount and receives a set amount of tournament chips.

Hit and Run - Refers to when a player sits down at an online table and win's a hand early in the game and then leaves instantly with his/her profit. This is seen as bad table etiquette in online games and in frowned upon in the OPS.

Solid - Refers to a good player e.g. he is a solid player

Grinder - Refers to an online player whom plays a large volume of online hands in a methodical way and makes a small profit each time they play i.e. to grind out a win.

Pooh-Bah - A pompous ostentatious official, especially one who, holding many offices, fulfills none of them.

Railbirds - Refers to the players which watch online games regularly

Railing - The process whereby one watches online poker games one is not involved in. Very popular when sponsored pro's such as Tom Dwan are playing online

Fold Equity - A term used to weigh up that if you fold a hand that you will be right a percentage of times. Obviously the higher your equity in a situation the more you should fold.

VPIP (Voluntary Put In Pot) - The amount of times a player will put money into the pot voluntarily i.e. calling a bet when not in the big or small blind. Often referred to and used in percentage terms

Plus/Negative EV (Expected Value) - A complicated poker term that uses maths to determine the expected value a player has in a poker game. If a player has a Plus EV and still loses money in a poker session it would then suggest that he/she was unlucky or variance was against them. 
Table 1 - The Online Poker Ecosystem

\author{
Online Poker Sites \\ Pokerstars.com - The most popular online poker room throughout the world. \\ Party Poker.com - An emerging online site which is owned by the Partygaming Corporation. \\ 888.com - Popular international online poker site \\ Betfairpoker.com - Popular sports betting site with an ever increasing online poker site.
}

\title{
Online Poker Forums
}

$\mathbf{2 + 2}$ - The most popular and well renowned online poker forum in the world. The site has over 250,000 members. It provides an arena for discussion, debate, co-operation and collaboration between online players.

Deuces Cracked - An online poker forum founded by online player Jay Rosenkrantz and home to thousands of avid online players who discuss the strategy of the game of poker.

High Stakes DB - Primarily a poker tracking site which tracks all the high limit poker games online and gives evidence of the winners and losers in those games, includes a popular online forum.

Cardrunners - An online poker training site, where online poker professionals give advice and training. However as with High Stakes DB, its forum has also gained in membership recently.

\section{Popular Magazine/Video Sites}

Cardplayer.com - Hugely popular online site and magazine which details current events, player blogs and interviews in the poker world.

Bluff Magazine - Magazine aimed at the poker community in particular avid followers of poker celebrities.

Pokertube.com - The YouTube of the poker world. Members upload videos on all poker related topics.

Pokernews.com - Poker reporting site which provides live coverage and updates on online and offline poker tournaments such as WCOOP and WSOP respectively, while also publishing player blogs, videos and articles related to poker events.

\section{Poker Reporting/Tracking Sites}

PokerTableRatings.com - A poker tracking website which tracks online player's play/results across popular online poker sites. Therefore any online player can be searched to see their winnings/loses, stakes they play, frequency of play, aggression levels within play etc.

Pokerlistings.com - Tracks player traffic on most online sites, and also provides players with information on poker sites and the various offers they provide.

Sharkscope.com - Poker tracking website which tracks the results of online players play/results specifically dealing with tournament and sit and go formats of poker.

* The platforms/sites chosen in Table 1 were chosen on the basis of their renowned status, and popularity amongst forum participants involved in the online poker ecosystem. 


\section{Figure 1 - The Influence of the OPS}

\section{Traditional Play}

Only enter a pot with premium hands

Check-raising - an extreme sign of strength

Lots of Limpers pre-flop

Game predominantly based on read \& feel

Conventional Language

\section{Evolution of Poker Play}

“Any two" style of play

Check-raising the norm

Common place to $3 / 4 / 5$ bet pre flop

Game predominantly based on maths and equity

Emerging/Ever-Changing Language 
Figure 2 - Player Dialogue Demonstrating Collaboration and Cooperation within OPS

Forum Member 2+2, Section: Micro Stakes NL ${ }^{1}$

Re: how do I play this against unknown???

PokerStars $\$ 0.10 / \$ .025$ No Limit Hold'em - 6 Players

Pre-Flop: (\$0.35) Hero is in MP with $K$ of spades, $K$ of clubs

1 fold, Hero raises to $\$ 1, \mathrm{CO}$ calls $\$ 1$, BTN calls $\$ 1,1$ fold, BB calls $\$ 0.75$

Flop: (\$4.10) 9 of diamonds, $T$ of hearts, 4 of diamonds

BB checks, Hero bets $\$ 3.10$, CO folds, BTN folds, BB raises to $\$ 10$, Hero requests TIME?????

\section{REPSONSE}

Respondent A: You get 1:2.5 on your money after his all in. Definitely call. Most probably it is a draw. If it's a set or two pair... LAG's also has to get paid from time to time

Respondent B: I'd probably go ahead and get it in, watch him flip over QJ, KT of diamonds and then throw something when the 8 of diamonds binked on the turn. In my opinion, this can be a lot more than something that has you crushed. It could even be an AT off trying to "protect his hand".

Respondent C: yeah you're committed on a draw heavy board, get it in

\footnotetext{
${ }^{1}$ Refer to appendix for decipher of language used
} 
Figure 3 - Player Dialogue Demonstrating Competition and Hierarchy within the OPS

Forum Member 2+2, Section; Micro Stakes PL/NL

Re: NL50; Floating in bvb

PartyPoker $\$ 0.25 / \$ 0.50$ No Limit Holdem

Pre-Flop: (\$0.75, 5 Players) Hero is BB with 9 of hearts, $T$ of hearts

3 folds, SB raises to $\$ 2$, Hero calls $\$ 1.50$

Flop: $Q$ of spades, 7 of hearts, 6 of diamonds ( $\$ 4,2$ players)

SB bets $\$ 2.50$, Hero calls $\$ 2.50$

Turn: $\mathrm{J}$ of hearts ( $\$ 9,2$ players)

SB bets $\$ 6$, Hero raises to $\$ 15, \mathrm{SB}$ calls $\$ 9$

River: 9 of diamonds

SB checks, Hero checks $(\$ 39,2$ players)

Final Pot: \$39

Hero shows: 9 of hearts, $\mathrm{T}$ of hearts

SB shows: J of diamonds, Q of diamonds

SB wins $\$ 37.05$

Hero lost \$19.50

RESPONSE

Respondent A: so why do we raise turn

Respondent B: yay let's raise our double-gs $+f d$ and get into a high variance spot instead of seeing river

Respondent C: Raising the turn and not firing the river makes little sense all the hands we fold on the turn will check/fold the river most often anyways. Plus his turn bet is small and there are implied because he won't put us on backdoor hearts etc.

$H$ Hever folds a $Q$ on the turn, unlikely he ever folds it on a river blank either. So call turn in my opinion. 
Figure 4-Roles and Titles within OPS - 2+2 Forum Post Titles

\begin{tabular}{|cc|}
\hline Title & No of Posts Required \\
Stranger & $0-15$ \\
Newbie & $15-50$ \\
Enthusiast & $50-100$ \\
Centurion & $100-200$ \\
Journeyman & $200-400$ \\
Grinder & $400-700$ \\
Adept & $700-1200$ \\
Old Hand & $1200-2000$ \\
Veteran & $2000-3500$ \\
Pooh-Bah & $3500-6000$ \\
Carpal \'Tunnel & $6000+$ \\
& \\
\hline
\end{tabular}


Figure 5 - Player Dialogue Demonstrating Player Enhancement within Hierarchy

Forum Member Deuces Cracked, Section: Medium Stakes
Re: Did I play this badly??
Pokerstars $\$ 5 / 10$ Omaha Cash Games - 6 Players
Pre-Flop: Hero is in UTG+1 with 3 of diamonds, K of diamonds, J of clubs, 4 of
clubs

(1 folds), Hero raises to $\$ 35$, (3 folds), BB calls $\$ 25$

Flop: (\$75) $Q$ of diamonds, 5 of hearts, $T$ of diamonds

BB checks, Hero bets $\$ 72, \mathrm{BB}$ raises to $\$ 288$, Hero calls $\$ 215$ and is all-in

Turn: (\$649) 4 of hearts

River: (\$649) A of spades

\section{Results:}

BB showed: 9 of hearts, $\mathrm{K}$ of clubs, 9 of clubs, $\mathrm{T}$ of hearts

Hero showed: 3 of diamonds, $\mathrm{K}$ of diamonds, $\mathrm{J}$ of clubs, 4 of clubs

\section{Hero wins \$646}

\section{RESPONSE}

Respondent A: dude are you serious?!!!

Respondent B: Just another, did I play this badly brag post. It should be entitled "Look everyone I won a $\$ 600$ pot". What is it with all these newbies with no clue

Respondent C: this is absolutely ******* standard!!!! why did you post this?

Respondent D: Luck box braggin about being a luck box.... good job. Prob broke soon or already

Respondent E: The whole thing is a joke. PTR confirms that it was a real hand. The kid had never played over .5/1 and then for giggles tried 5/10. He hit his big hand and left after 7 minutes. It's a standard hit and run. What he won't tell you is that he next played $.25 / .5$ and lost \$11.50. Please ban this clown. I don't even believe that he made me feel compelled to respond to his post. 